\title{
Superconductive Junctions with Trappinglayers for the Detection of X-rays
}

\author{
J.B. le Grand, M.P. Bruijn, M. Frericks, W. Laauwen, D. Nilsson, \\ P.A.J. de Korte, P. Valko*, J.G. Gijsbertsen**, E.P. Houwman** \\ and J. Flokstra** \\ Laboratory for Space Research, Sorbonnelaan 2, 3584 CA Utrecht, the Netherlands \\ * On leave from Department of Physics, Slovak Technical University, Ilkovic̀ova 3, \\ 812-19, Bratislava, Slovakia \\ ** University of Twente, Faculty of Applied Physics, Low Temperature Group, P.O. Box \\ 217, 7500 AE Enschede, the Netherlands
}

\begin{abstract}
Addition of a trapping layer to an SIS junction improves its performance as an $X$ ray detector. In this article $X$-ray induced pulse height and decay time spectra will be presented as a function of bias voltage. These measurements are in good agreement with a description based on the time constants for trapping, excitation and tunneling calculated by means of a model for proximity layers developed by Golubov et al.. ${ }^{1,2}$ The interpretation of the data doesn't require an initial fast loss process for the created quasi-particles as discussed by Van Vechten. ${ }^{3}$
\end{abstract}

\section{Introduction}

Giaever-type junctions have the potential to become high energy resolution Xray detectors. The energy resolution is ultimately limited by the Poisson statistics on the number of quasi-particles created by the absorbed X-ray photon which leads to a resolution of a few eV for X-rays in the $0.1-10 \mathrm{keV}$ energy range. For $5.89 \mathrm{keV}$ however, the best results achieved are $41 \mathrm{eV}$ for a tin junction ${ }^{4,5}$ and $88 \mathrm{eV}$ for a $\mathrm{Nb} / \mathrm{Al}$ junction. ${ }^{6}$

Since part of this discrepancy is due to quasi-particle loss mechanisms, we have added an aluminium trapping layer in between the $\mathrm{Nb}$ base electrode and the tunnel barrier. The presence of this layer creates a quasi-particle storage volume, that is not in contact with the detector edges and surfaces and their associated loss processes. ${ }^{3,7,8}$ Because this layer is thin, the quasi-particle tunnel rate is considerably enhanced, which in itself reduces the influence of the loss processes. 


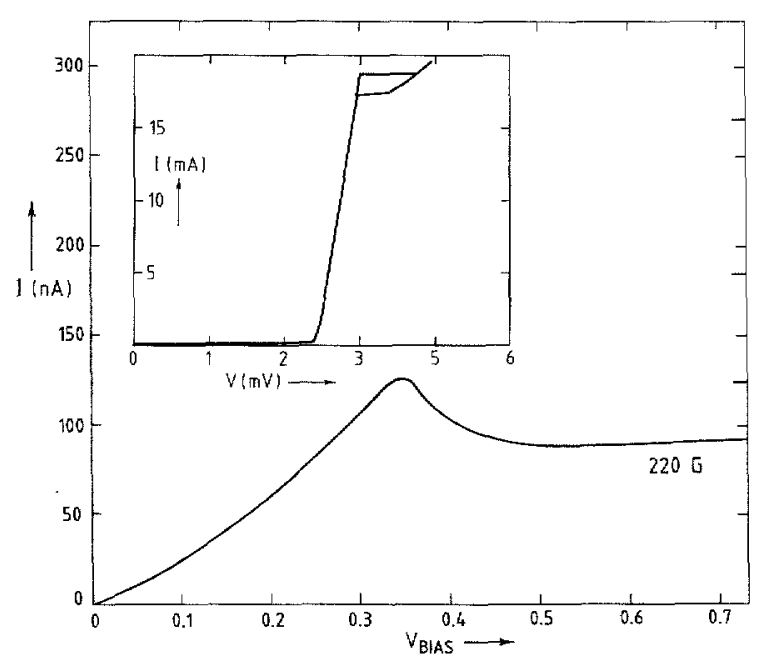

Fig. 1. I/V-curve measured at $220 \mathrm{G}$. The insert shows the $\mathrm{I} / \mathrm{V}$ curve and the main figure the thermal part of the subgap current after subtraction of the leakage current.

\section{Experimental Set-up}

The junction investigated is produced by sputtering onto an oxidized silicon substrate. The base electrode consists of $300 \mathrm{~nm} \mathrm{Nb}$ and a $25 \mathrm{~nm}$ Al trapping layer. The oxide barrier on top of this is corered by $3 \mathrm{~nm} \mathrm{Al}, 195 \mathrm{~nm} \mathrm{Nb}$ and $22 \mathrm{~nm} \mathrm{Al}$, which form together the counter electrode. The barrier size equals $100 \times 100 \mu \mathrm{m}^{2}$ and has a current density of $100 \mathrm{~A} / \mathrm{cm}^{2}$.

The junction is cooled down in a ${ }^{4} \mathrm{He}$-cryostat with a base temperature of 1.15 $\mathrm{K}$, and a superconducting magnet is used to suppress the Josephson current and Fiske steps.

In front of the junction a radioactive ${ }^{55} \mathrm{Fe}$ source is mounted, which irradiates the junction with $5.89 \mathrm{keV} \mathrm{MnK}_{\alpha}(88 \%)$ and $6.49 \mathrm{keV} \mathrm{Mn} \mathrm{K}_{\beta}(12 \%)$ X-rays. The first amplification of the signal takes place in the cryostat by a cold preamplifier $(120 \mathrm{~K})$. Pulse analyzing electronics outside the cryostat determine the pulse height and the decay time of the signal.

\section{I/V-curves}

The $\mathrm{I} / \mathrm{V}$ curve measured at $1.15 \mathrm{~K}$ is presented in the insert of figure 1 for $\mathrm{B}=$ $220 \mathrm{G}$. The voltage at which the current increase sets in, corresponds to the sum of the bandgaps of the two films, facing the barrier and equals $2.39 \mathrm{meV}$. At $T=1.15$ $\mathrm{K}$ and $T=1.25 \mathrm{~K}$ detailed measurements of the subgap have been carried out. At $\mathrm{T}=1.15 \mathrm{~K}$ the subgap current is dominated by a temperature independant leakage current. By subtraction of this leakage current from the measured subgap current at $1.25 \mathrm{~K}$, the current caused by tunneling of thermal quasi-particles is obtained. 
The result is shown in figure 1 . The voltage at which this tunneling current shows a maximum equals $0.33 \mathrm{meV}$ and corresponds to the bandgap difference of the base and counter electrode.

From these two sets of measurements the bandgaps of both electrodes are determined to be $\Delta_{1}=1.36 \mathrm{meV}$ and $\Delta_{2}=1.03 \mathrm{meV}$. The bandgap $\Delta_{2}$ of the base electrode is strongly reduced with regard to the bulk bandgap for $\mathrm{Nb}\left(\Delta_{\mathrm{Nb}}=1.5\right.$ $\mathrm{meV}$ ) due to proximity effect. Also $\Delta_{1}$, the bandgap of the counter electrode is slightly reduced. This is not due to the proximity effect because the Al protection layer on top of the barrier is too thin to cause such a reduction. We assume that it is caused by the fact that the $\mathrm{Nb}$ of this layer is sputtered on top of aluminium and has therefore a degraded crystal structure. Bandgap measurements on junctions with a thin Al-layer as part of the base electrode $\left(\Delta_{1}+\Delta_{2}=2.84 \mathrm{meV}\right)$ indicate that the bandgap of $\mathrm{Nb}$ in the base electrode, made by our process, is reaching about the bulk value.

From a theoretical point of view the reduction of the bandgaps in both electrodes can be descibed by the proximity parameters $\gamma_{M}$ and $\gamma_{B} \cdot{ }^{1} \gamma_{B}$ is related to the transparency of the interface between the two materials which are in proximity. For $\mathrm{Nb} / \mathrm{Al}$ the interface transparency is very large, ${ }^{9}$ so $\gamma_{B}=0 . \gamma_{M}$ is related to the bandgap suppression by the Al-layer. From the measured bandgaps of our films we can compute $\gamma_{M, \text { base }}=0.82$ and $\gamma_{M \text {,counter }}=0.20 .^{2}$

\section{X-ray results}

Typical X-ray results are shown in figure 2, where the pulse height and decaytime for each individual X-ray event is indicated by a point. The cloud of datapoints at the right part of the diagram is due to testpulses, used for the calibration of pulse height and decaytime. The other two, connected, clouds are due to X-rays absorbed in the base and in the counter electrode. To get more information on the pulse height, pulse height spectra are made. In figure 3 a pulse height spectrum derived from the data shown in figure 2 is given.

From similar data sets, accumulated at different bias voltages, we have derived the maximum amplitude for $5.89 \mathrm{keV} \mathrm{MnK}_{\alpha} \mathrm{X}$-rays for both peaks and their corresponding signal decay times. By application of the proper calibration data for pulse height and decay time circuits these values can be expressed in electrons and microseconds, respectively. In figure 4 the pulse heights and decay times are given as a function of bias voltage for the higher and the lower peak in the spectrum. From the measurents it is clear that the highest peak in the spectrum shows a clear maximum for a bias voltage around $\Delta_{1}-\Delta_{2}$. For the lowest peak this is not seen. From theoretical tunneltime analysis it is clear that the signal from the electrode with the lowest bandgap is expected to show such a maximum, while the signal from the electrode with the highest bandgap does not. For this reason we identify the highest peak in the spectrum to X-ray events absorbed in the base electrode. This result is confirmed by the measured intensity in both peaks.

In this article the interpretation of the base electrode is presented. From the counterelectrode hardly any signal was expected due to the Al-cover layer, which was assumed to trap the quasi particles away from the barrier. The origin of the considerable signals found experimentally for the counter electrode is not fully un- 


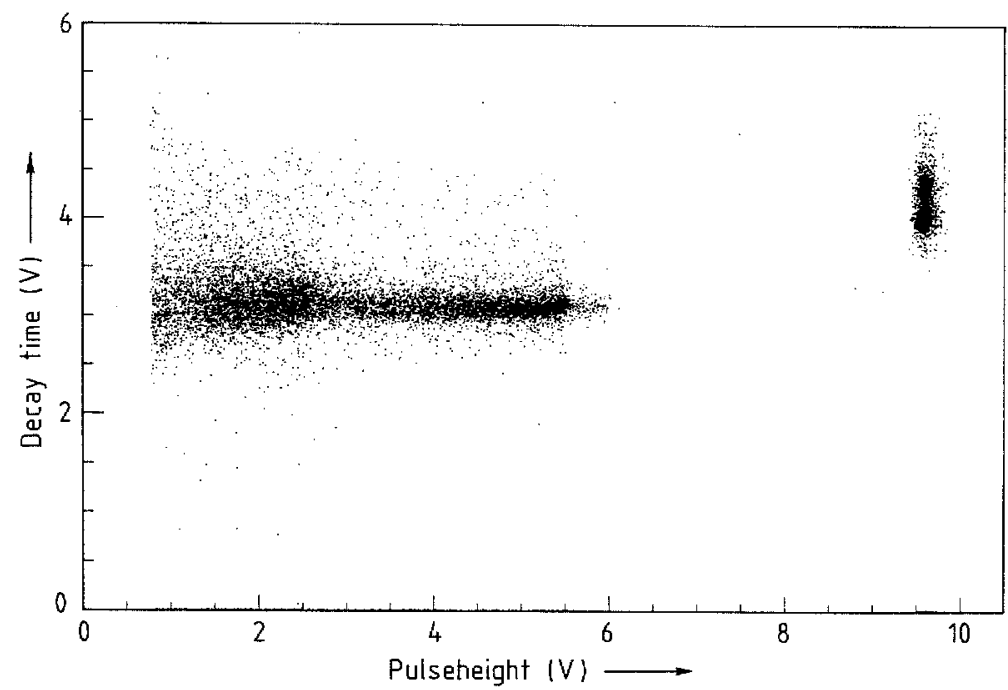

Fig. 2. Pulse height versus decay time for ${ }^{55} \mathrm{Fe} X$-rays measured at $\mathrm{B}=220 \mathrm{G}$ and $V_{b}=.5 \mathrm{mV}$.

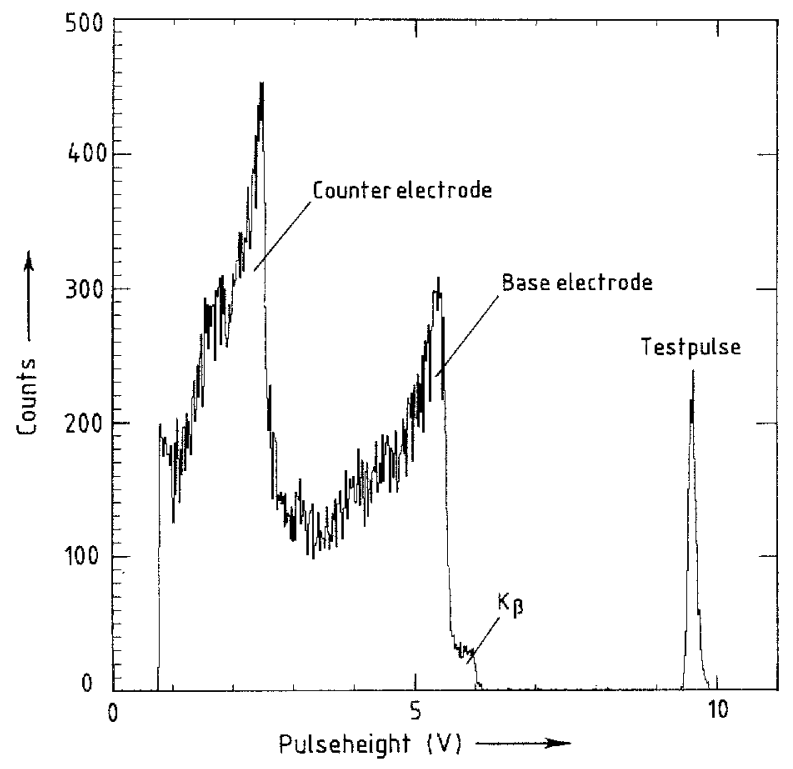

Fig. 3. Pulseheight spectrum of ${ }^{55} \mathrm{Fe} \mathrm{X}$-rays measured at $B=220 \mathrm{G}$ and $V_{b}=.5$ $\mathrm{mV}$. 

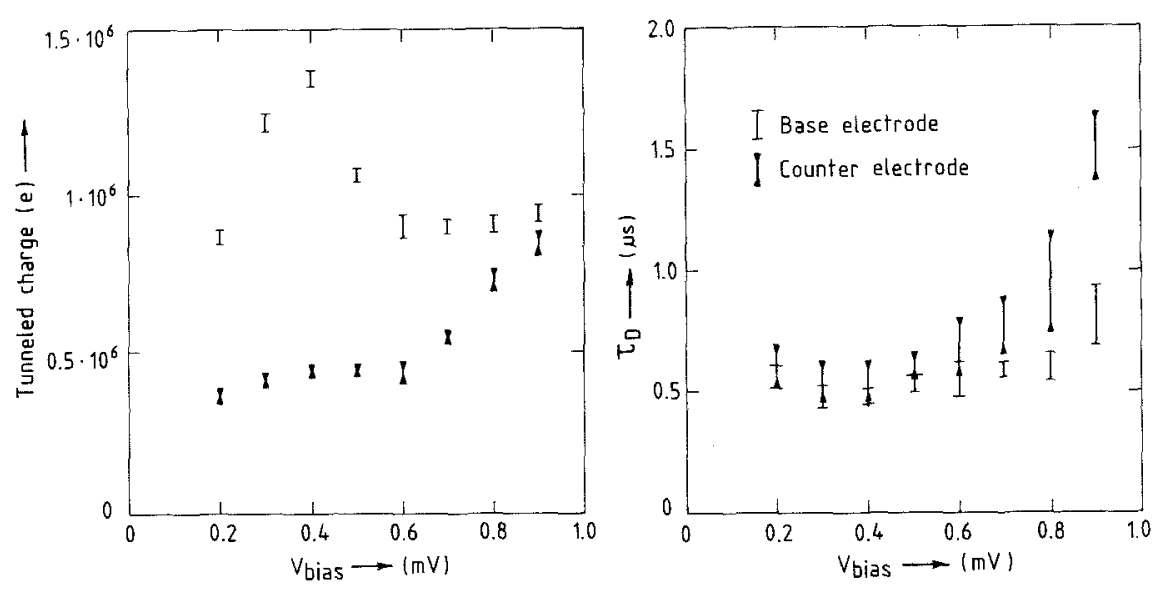

Fig. 4. $\operatorname{MnK}_{\alpha}(5.89 \mathrm{keV})$ pulse height for the highest and the lowest peak in the spectrum as a function of bias voltage (left picture) and decay time for $\mathrm{MnK}_{\alpha}$

$(5.89 \mathrm{keV}) \mathrm{X}$-rays for the highest and the lowest peak in the spectrum as a function of bias voltage (right picture).

derstood, although the bandgap reduction of the counter electrode near the barrier might form part of the explanation. The signals from the counter electrode are, however, used to correct the signals of the base electrode for back tunneling.

\section{Quasi-particle processes in the base electrode}

The energy of the X-ray photon $E_{p h}$, absorbed in one of the superconductive layers of the junction, initiates a cascade of quasi-particles and phonons. On a timescale of typically a few nanoseconds the quasi particles and phonons relax to energies just above $\Delta$ and $2 \Delta$, respectively. At that stage the number of quasiparticles created equals: ${ }^{10}$

$$
N_{0}=\frac{E_{p h}}{\epsilon} \approx \frac{E_{p h}}{1.7 \Delta}
$$

In this formula the value of $\Delta$ will be in between those for the trapping layer and the bulk $\mathrm{Nb}$.

The quasi-particles in the trap and the bulk subsequently undergo various processes, which are summerized in figure 5. The transport of quasi-particles in and out of the trapping layer is dominated by four processes, three of which can be quantitatively determined from the $\gamma_{M}$-value. ${ }^{1,2}$ Quasi-particles in the bulk of the base electrode get trapped in the trapping layer with a time constant $\tau_{\text {trap }} \approx 12 \mathrm{~ns}$. Quasi-particles in the trap can get thermally excited into the bulk, a process governed by the excitation time constant $\tau_{e x c} \approx 84 n s$. Furthermore the quasi-particles can also leave the trap through tunneling to the counter electrode, characterized by $\tau_{\text {tun }} \approx 3 \mu \mathrm{s}$. The fourth transport process of quasi-particles from the trap is by a 


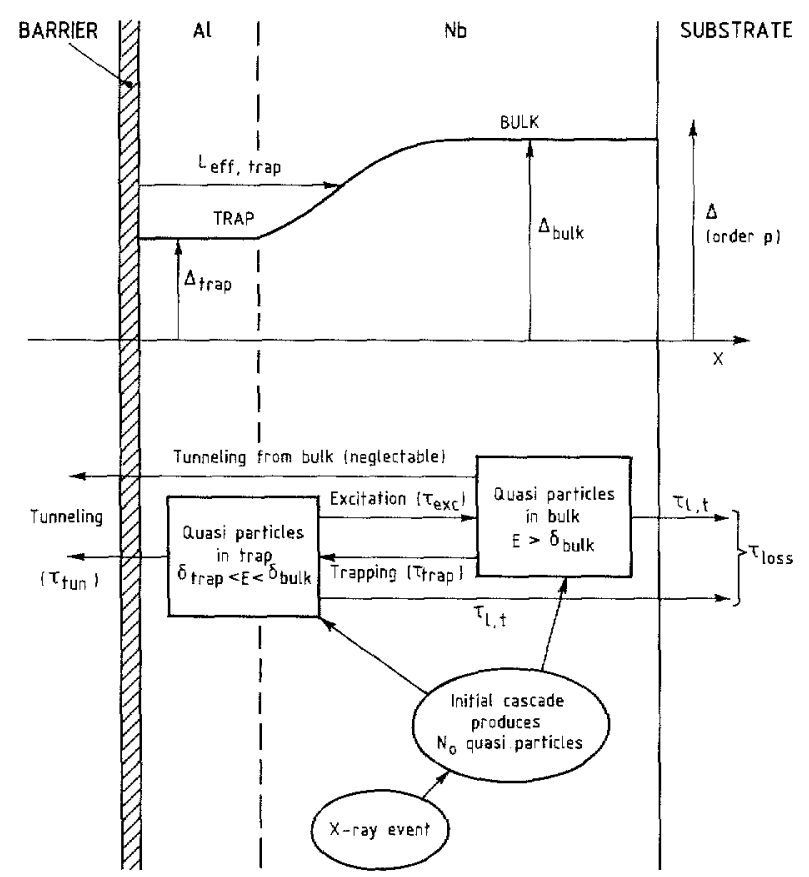

Fig. 5. Schematic diagram of quasi-particle processes in the base electrode.

loss process characterized by the loss time constant from the trap $\tau_{l, t}$. This process, however, is expected to be very slow since the trapping layer is not in contact with any surface, except for the tunnel barrier.

The transport processes of quasi-particles towards and from the Nb bulk, not discussed already in the alinea above, are tunneling to the counter electrode and loss processes. The time constant for tunneling to the counter electrode is rather long given the layer thickness of the base electrode and the current density of the barrier, i.e. approximately $15 \mu \mathrm{s}$. Therefore this process can be neglected. The loss time from the bulk $\tau_{l, b}$ is most likely dominated by trapping to the $\mathrm{Nb} / \mathrm{SiO}_{2}$ interface of the base electrode and the silicon wafer as well as to more localized areas with a reduced bandgap due to penetration of the magnetic field into the base electrode.

If we neglect tunneling from the bulk the chance $P_{b}$ that a quasi-particle in the base electrode tunnels to the counter electrode equals:

$$
P_{b}=\frac{\Gamma_{\text {tun }}}{\Gamma_{\text {tun }}+\Gamma_{\text {loss }}}=\frac{1}{1+\frac{\tau_{\text {tun }}}{\tau_{\text {loss }}}}
$$

where $\Gamma_{t u n}$ and $\tau_{\text {tun }}$ are for the trap and $\Gamma_{\text {loss }}$ and $\tau_{\text {loss }}$ are the combined values for trap and bulk. Because the chance for back tunneling from the upper electrode to the base electrode is small $(\approx 12 \%$ ) the decay time of the signal from the base electrode can approximately be described by the time constant $\tau_{D}$ which is given 

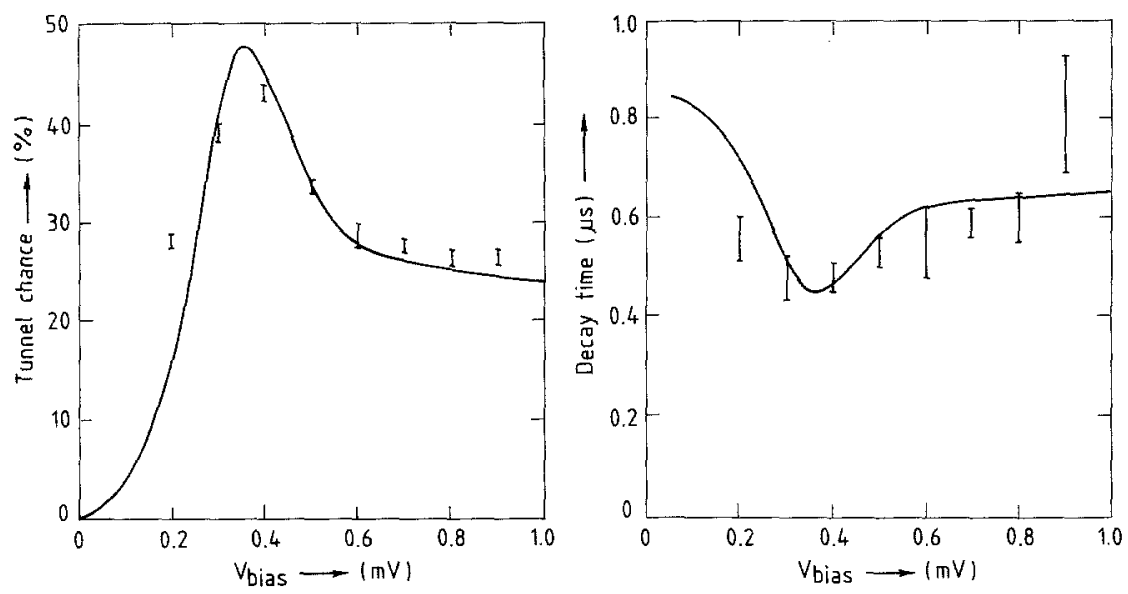

Fig. 6. Results for a fit of the experimental pulse heights (left) and decay times (right) with $N_{0}=2.7 * 10^{6}$ and $\tau_{\text {loss }}=0.86 \mu s, \mathrm{~B}=220 \mathrm{G}$

by:

$$
\frac{1}{\tau_{D}}=\frac{1}{\tau_{\text {tun }}}+\frac{1}{\tau_{\text {loss }}}
$$

\section{Data interpretation}

In order to compare the data with theory we correct them for back tunneling and subsequently express them as a tunnel chance $P_{b}$ for the initially created number of quasi-particles $N_{0}$. Simple arguments result in:

$$
P_{b}=\frac{Q_{b}}{Q_{c}+e N_{0}}
$$

with $Q_{b}$ and $Q_{c}$ the measured tunneled charge for events in the base and counter electrode, respectively. In this formula it is assumed that the initial charge $e N_{0}$ is equal in base and counter electrode, which might not be totally true given the slightly different bandgaps in both electrodes.

Now, the measured decay time $\tau_{D}$ and the tunnel chance $P_{b}$ derived from the data by means of formula 4 can be fitted to the theoretical tunnel chance and decay time as defined in formulas 2 and 3 . In this combined fit, obtained by reduced $\chi^{2}$-analyses, the only free parameters are the loss time constant in formulas 2 and 3 and the initial number of quasi-particles $N_{0}$ in formula 4 , since all other time constants are defined by measured $\gamma_{M}$-values and Golubov. ${ }^{1,2}$

In figure 6 is shown that in case of $B=220 G$ a nice fit of pulse heights and decay times is obtained for $N_{0}=2.7 \pm 0.3 * 10^{6}$ and $\tau_{\text {loss }}=0.86 \pm 0.14 \mu \mathrm{s}$.

Support for the correctness of this data interpretation is given by the fact that the loss time derived by means of formulas 2,3 and 4 is independant of bias voltage $\left(\chi_{r}^{2}=1.36\right)$. This is in agreement with expectations. 
It is important to realize that these data cannot be fit with the proximity model of McMillan. ${ }^{12}$ Tunnel times calculated with this model would have been about a factor 5 shorter, while the trapping times would have been much longer. Such time constants would not fit with the bias voltage dependance of the signal we measured.

\section{Conclusions}

- The model based on the theory developed by Golubov et al. ${ }^{1,2}$ gives a good quantitative description of the experimental X-ray results as a function of bias voltage. It may therefore succesfully be applied for modelling the trapping process in absorber junction combinations as proposed by Booth and Kraus. ${ }^{14,15}$

- The measurements indicate that initial number of quasi-particles $N_{0}$ is in good agreement with theory. ${ }^{10}$ This eliminates the hot spot model ${ }^{11}$ for $\mathrm{Nb} / \mathrm{Al}$ junctions as an explanation for the fact that these junctions, used as $\mathrm{X}$-ray detectors did not give the energy resolution and pulse height theoretically expected.

- In case one attributes the measured loss time, $0.86 \mu \mathrm{s}$, to loss in the Nb-bulk of the base electrode, this loss time equals $120 \mathrm{~ns}$, in agreement with former measurements on junctions with thin Al-layers (80ns). ${ }^{13}$

\section{Acknowledgements}

The investigations form also part of the program of the Foundation for Fundamental Research on Matter (FOM) and have been supported by the Netherlands Technology Foundation (STW).

\section{REFERENCES}

1. A.A. Golubov and M.Y. Kupriyanov, Sov. Phys. JETP $\underline{69}, 805$ (1989)

2. Golubov et al., to be published in Phys.Rev.B (1993)

3. D. Van Vechten and K.S. Wood, Phys.Rev.B43, 12852 (1991)

4. D. Twerenbold, Phys.Rev.B34, 7748 (1986)

5. D. Twerenbold, Europhys.Lett. 1,209 (1986)

6. M. Kurakado et al, proc. of SPIE's symp. on EUV, X-ray, and Gamma-Ray Instrumentation for Astronomy III, Volume 1743, 351, San Diego (1992)

7. J. Halbritter, Appl.Phys.A. 4주, 1 (1987)

8. P.A.J. de Korte, Proceedings of the ESA symposium on photon detectors for space instrumentation, ESA SP-356, 41, Noordwijk (1992)

9. E.P. Houwman et al. IEEE Trans. on Appl.Superc. $\underline{3}, 2170$ (1993)

10. M. Kurakado, Nucl.Instr.and Meth.A196, 275 (1982)

11. D.J. Goldie et al. invited paper presented at SQUID'91, Berlin (1991)

12. W.L. McMillan, Phys.Rev.175, 537 (1968)

13. J.B. le Grand et al. IEEE trans.on Appl.Superc. 3 , 2070 (1993)

14. N.E. Booth, Appl.Phys.Lett.50, 293 (1987)

15. H. Kraus et al., Phys.Lett.B231, 195 (1989) 